\title{
Timosaponin derivative YY-23 acts as a non- competitive NMDA receptor antagonist and exerts a rapid antidepressant-like effect in mice
}

\author{
Qi ZHANG, Fei GUO, Zhi-wen FU, Bing ZHANG, Cheng-gang HUANG* ${ }^{*}$ Yang LI*
}

Key Laboratory of Receptor Research, Shanghai Institute of Materia Medica, Chinese Academy of Sciences, Shanghai 201203, China

\begin{abstract}
Aim: $N$-methyl-D-aspartic acid (NMDA) receptor modulators have shown promising results as potential antidepressant agents, whereas timosaponins extracted from the Chinese herb Rhizoma Anemarrhenae exhibit antidepressant activities. In the present study we examined whether YY-23, a modified metabolite of timosaponin B-III, could affect NMDA receptors in rat hippocampal neurons in vitro, and evaluated its antidepressant-like effects in stressed mice.

Methods: NMDA-induced currents were recorded in acutely dissociated rat hippocampal CA1 neurons using a whole-cell recording technique. C57BL/6 mice were exposed to a 6-week chronic mild stress (CMS) or a 10-d chronic social defeat stress (CSDS). The stressed mice were treated with $Y Y-23\left(20 \mathrm{mg} \cdot \mathrm{kg}^{-1} \cdot \mathrm{d}^{-1}\right)$ or a positive-control drug, fluoxetine $\left(10 \mathrm{mg} \cdot \mathrm{kg}^{-1} \cdot \mathrm{d}^{-1}\right)$ for $3 \mathrm{weeks}$. Behavioral assessments were carried out every week.

Results: In acutely dissociated rat hippocampal CA1 neurons, YY-23 selectively and reversibly inhibited NMDA-induced currents with an $\mathrm{EC}_{50}$ value of $2.8 \mu \mathrm{mol} / \mathrm{L}$. This inhibition of NMDA-induced currents by YY-23 was non-competitive, and had no features of voltagedependency or use-dependency. Treatment of the stressed mice with YY-23 not only reversed CMS-induced deficiency of sucrose preference and immobility time, and CSDS-induced reduction of social interaction, but also had faster onset as compared to fluoxetine. Conclusion: YY-23 is a novel non-competitive antagonist of NMDA receptors with promising rapid antidepressant-like effects in mouse models of CMS and CSDS depression.
\end{abstract}

Keywords: Rhizoma Anemarrhenae; timosaponin; YY-23; depression; NMDA receptor antagonist; chronic mild stress; chronic social defeat stress; fluoxetine

Acta Pharmacologica Sinica (2016) 37: 166-176; doi: 10.1038/aps.2015.111; published online 21 Dec 2015

\section{Introduction}

The incidences of psychiatric diseases preceded by depressive disorder have been rising annually as the pressure of life in modern society grows. In response, the development of antidepressants has been ongoing for decades ${ }^{[1]}$. Tricyclic antidepressants and monoamine oxidase inhibitors were the earliest two types of antidepressants developed, represented by imipramine and iproniazid, respectively, and are still widely used clinically. According to the mechanism of their antidepressant-like effects, the 'monoamine deficiency hypothesis' holds the view that a deficiency of synaptic monoamines (eg, dopamine, serotonin and norepinephrine) was the main cause of depression ${ }^{[2]}$. However, the clinical application of these agents was limited because of their side effects, which

\footnotetext{
* $T o$ whom correspondence should be addressed.

E-mail liyang@simm.ac.cn (Yang LI);

cghuang@simm.ac.cn (Cheng-gang HUANG)

Received 2015-07-08 Accepted 2015-10-28
}

resulted from poor selectivity; therefore, selective serotonin reuptake inhibitors (SSRIs) became the main therapeutic drugs that were widely applied in clinical settings. The most common SSRI is fluoxetine, which has been widely reported to reverse or prevent the depressive-like behaviors induced by chronic mild stress $(\mathrm{CMS})^{[3,4]}$. However, the problems related to the slow onset, long timescale and low curative rate of these drugs have not been sufficiently resolved. Therefore, there is an urgent to elucidate the pathogenesis of depressive disease and to develop a new generation of antidepressants with rapid action and fewer side effects.

For the past few years, the emerging role of the $N$-methyl$D$-aspartic acid (NMDA) receptor in psychiatric diseases has gradually attracted the attention of a growing number of researchers. Evidence from post-mortem and in vivo brain imaging studies implicated abnormalities in glutamate signaling in patients with depression ${ }^{[5]}$. One of the breakthrough discoveries was the observation that the administration of an NMDA receptor antagonist, ketamine, produced a fast 
antidepressant-like effect ${ }^{[6,7]}$. Moreover, the underlying signaling pathways of ketamine also revealed novel targets for antidepressant discovery and development ${ }^{[8-11]}$. Several NMDA receptor modulators have been developed and currently show promise as potential antidepressant agents with favorable effects and rapid onset in both clinical settings and animal models of depression ${ }^{[5]}$. The results from these ketamine studies and the preclinical reports on a variety of other agents acting on the NMDA receptor have aroused increasing interest in the development of NMDA modulators as novel therapeutic agents to treat mood disorders ${ }^{[12,13]}$. The combination of NMDA receptor antagonists with first-line antidepressants has the potential to become a more effective and efficient therapeutic strategy for treatment-resistant patients or those with suicidal tendencies ${ }^{[14,15]}$. Although its beneficial effects were promising, the clinical use of ketamine has been hampered by its psychotomimetic character and toxicity due to drug abuse ${ }^{[16]}$.

The psychotropic effects of natural products have been of interest to many clinicians and scientists due to their lack of side effects and toxicity ${ }^{[17,18]}$. Compelling research studies have shown that many types of effective components from natural products were effective agents against central nervous system diseases. For example, huperzine A and l-stepholidine (l-SPD), which have been proven to be efficient at reversing cognitive deficits, appear to be good candidates for the treatment of Alzheimer's disease and schizophrenia, respectively ${ }^{[19,20]}$. Recent research suggests that timosaponin, which is derived from Rhizoma Anemarrhenae, has unique sedative action on mice with neural dysfunction caused by stress. Many preclinical studies also indicated that timosaponin may have the potential to play an essential role in both depression and anxiety treatment ${ }^{[18,21]}$. Specifically, timosaponin B-II and B-III are the two main bioactive constituents that are thought to improve learning and memory. Moreover, B-III and its derivatives were reported to be important for the main antidepressive activity of timosaponin ${ }^{[22,23]}$. Although its detailed mechanism of action remains unknown, the therapeutic potential of timosaponin is obvious, and its mechanism of psychotropic action deserves further exploration.

We have found that there were several metabolites of B-III present in the brain areas that are related to mood disorders after intragastric administration of B-III in both mice and rats. A structurally modified derivative of one of the primary metabolites, YY-23 (Figure 1), elicited significant improvements in stress-related behaviors in our preliminary study ${ }^{[24]}$. In the present study, we used electrophysiological methods combined with behavioral studies to explore the psychotropic mechanisms of YY-23.

\section{Materials and methods Animals}

All Sprague-Dawley rats and C57BL/ 6 mice used in our experiments were purchased from Shanghai SLAC Laboratory Animal Co Ltd and kept in the animal center of the Shanghai Institute of Materia Medica at the Chinese Academy of

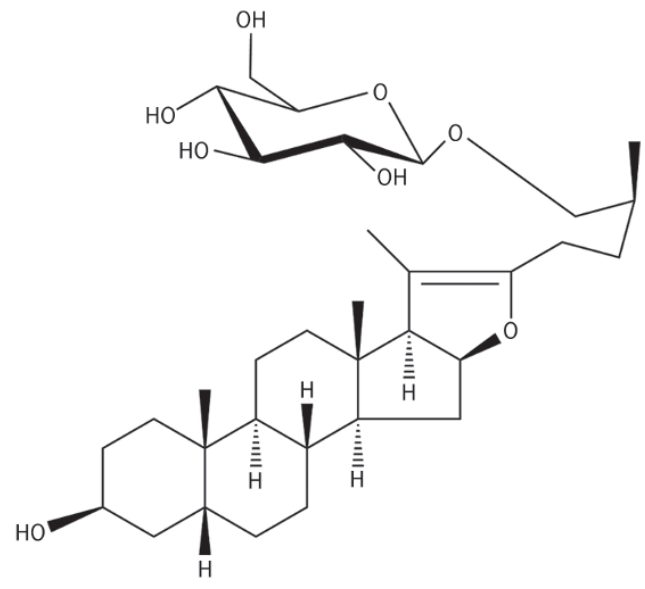

Figure 1. The chemical structure of YY-23.

Science (SIMM, CAS). The protocols were approved by the Institutional Animal Care and Use Committee, and the experiments were carried out in accordance with the EU Directive 2010/63/EU on the protection of animals used for scientific purposes.

\section{NMDA-current recording}

\section{Preparation of dissociated hippocampal neurons}

Experiments were carried out according to methods previously described ${ }^{[25,26]}$, with slight modifications. In brief, dissociated hippocampal neurons were prepared from 10-14-dold Sprague-Dawley rats. The whole brain was removed and dissected in oxygenated ice-cold dissociated solution that consisted of the following (in mmol/L): $\mathrm{Na}_{2} \mathrm{SO}_{4} 82, \mathrm{~K}_{2} \mathrm{SO}_{4}$ $30, \mathrm{MgCl}_{2}$ 5, HEPES 10, NaPy 1 and glucose 20 ( $\mathrm{pH}=7.4$ with $\mathrm{NaOH})$. Hippocampal slices $(500 \mu \mathrm{m})$ were cut with a vibrator slicer (VT1000S, Leica, Germany), and the CA1 region was dissected out. The CA1 pieces were treated with dissociated solution containing $3 \mathrm{mg} / \mathrm{mL}$ protease XXIII (Sigma, USA) at $32^{\circ} \mathrm{C}$ for $8 \mathrm{~min}$, washed three times with the dissociated solution and then incubated in dissociated solution containing 1 $\mathrm{mg} / \mathrm{mL}$ bovine serum albumin (Sigma) and $1 \mathrm{mg} / \mathrm{mL}$ trypsin inhibitor type II-S (Sigma, USA) for $1 \mathrm{~h}$. Neurons were dissociated with a series of fire-polished Pasteur pipettes by waiting for the neurons to adhere to the bottom of the recording dishes and then replacing the solution with an $\mathrm{Mg}^{2+}$-free external solution that omitted $\mathrm{Mg}^{2+}$ and consisted of the following (in mmol/L): $\mathrm{NaCl} 140, \mathrm{KCl} 3, \mathrm{CaCl}_{2}$ 2, HEPES 10 and glucose 10 $(\mathrm{pH}=7.4$ with $\mathrm{NaOH})$. Pyramidal neurons with large pyramidal-shaped cell bodies and thick apical dendritic stumps were chosen for this study.

\section{Whole-cell voltage-clamp recording}

Patch pipets (tip resistance 6-8 M $\Omega$ ) were pulled with borosilicate glass pipets (SUTTER P-97, SUTTER INSTRUMENT Co, USA) and filled with internal solution containing the following (in mmol/L): $\mathrm{KCl}$ 140, HEPES 10, $\mathrm{CaCl}_{2} 1$ and EGTA 10 $(\mathrm{pH}=7.4$ with $\mathrm{KOH})$. Current responses were recorded under a whole-cell voltage-clamp configuration using an Axonpatch 
200B amplifier (Axon Instruments, USA). The membrane potential was held at $-60 \mathrm{mV}$, except when the voltage-dependency of YY-23 action was tested. The data were filtered at 1 $\mathrm{kHz}$ and then acquired online by a computer through an Axon DigiData-1440A interface using the Clampex 10.3 Data Acquisition Module (Molecular Devices, USA).

\section{Drug application}

NMDA, a-amino-3-hydroxy-5-methyl-4-isoxazolepropionic acid (AMPA), glycine and dizocilpine (MK-801) were purchased from Sigma. YY-23 was provided by the Department of Phytochemistry at the Shanghai Institute of Materia Medica, dissolved in dimethylsulfoxide (DMSO, Sigma) and then diluted to a series of concentrations with $\mathrm{Mg}^{2+}$-free external solution. The DMSO content was maintained below $0.1 \%$, except when the efficacy of $15 \mu \mathrm{mol} / \mathrm{L}$ YY-23 was tested, and the DMSO control was carried out on this occasion. Other drugs were directly dissolved in the $\mathrm{Mg}^{2+}$-free external solution. The solutions were applied to the recorded neuron through the Rapid Solution Changer (BioLogic RSC-200) with a 10-tube head; $50 \mu \mathrm{mol} / \mathrm{L}$ AMPA, or $100 \mu \mathrm{mol} / \mathrm{L}$ NMDA in the presence of $2 \mu \mathrm{mol} / \mathrm{L}$ glycine was administered to induce current responses. When tested, YY-23 was applied $10 \mathrm{~s}$ prior to and during NMDA and AMPA application.

\section{CMS induction and drug evaluation}

We used the same method described in our previous work ${ }^{[24]}$, according to the naturalistic rodent modeling procedure of depression reported in previous studies ${ }^{[27,28]}$. Briefly, each mouse, with the exception of the control mice, was caged alone and exposed to a series of chronic, unpredictable, mild stressors, including $4 \mathrm{~min}$ of cold swimming, $12 \mathrm{~h}$ of food/ water deprivation, $12 \mathrm{~h}$ of a soiled cage, $12 \mathrm{~h}$ of cage rotation, alteration of the normal light and dark cycle, and $12 \mathrm{~h}$ of white noise ( $65 \mathrm{~dB}$ SPL). These stressors were randomly applied every week to avoid habituation and maximize unpredictability. C57BL/ 6 male mice (weighing 20-25 g) were randomly divided into different groups depending on their treatment. Seven control mice (CON) were kept under standard conditions, in which they were caged together and intragastrically (ig) administered vehicle, $0.5 \%$ sodium carboxymethylcellulose (CMC-Na), as a solvent control from week 1 to week 3 . Seven CMS-induced mice (CMS) were maintained under CMS conditions, in which each mouse was exposed to chronic mild stressors, caged alone and administered vehicle, 0.5\% CMC$\mathrm{Na}$ (ig), from week 1 to week 3. Six YY-23-treated mice (YY23) were maintained under CMS conditions and administered $20 \mathrm{mg} / \mathrm{kg}$ YY-23 (ig) (YY-23 was suspended in 0.5\% CMC-Na) per day for the last 3 weeks. Six fluoxetine-treated mice (FLX) were maintained under CMS conditions and intraperitoneally (ip) administered $10 \mathrm{mg} / \mathrm{kg}$ fluoxetine (fluoxetine was dissolved in water) per day for the last 3 weeks.

\section{Tail suspension test (TST)}

We evaluated mice using the tail suspension test each week using the method described in a previous study ${ }^{[24]}$. Briefly, the mice were suspended by placing their tails on the edge of a shelf at a height of $80 \mathrm{~cm}$ above the floor for $6 \mathrm{~min}$. A blinded experiment was conducted to record the immobility time during the last $4 \mathrm{~min}$ of the testing period, excluding the first 2 min.

\section{Sucrose preference test (SPT)}

We evaluated the mice using a weekly sucrose preference test. The mice were allowed to choose freely between two bottles during $24 \mathrm{~h}$ : one bottle contained 1\% sucrose solution, and the other contained normal drinking water. After the first $12 \mathrm{~h}$, the position of each bottle was switched to prevent the effects of position preference. The two bottles were weighed before and after the test. Sucrose preference was calculated as follows: sucrose preference $(\%)=($ sucrose intake $(\mathrm{g}) \div($ sucrose intake $(\mathrm{g})+$ water intake $(\mathrm{g}))) \times 100$.

\section{Open field test (OFT)}

As previously described ${ }^{[29-31]}$, the total distance travelled and the walking speed of the mice in an open field test were measured as indicators of locomotor activity. The mice were placed into the arena $(40 \times 40 \times 35 \mathrm{~cm})$ and allowed to explore freely for $5 \mathrm{~min}$. Locomotor activity was recorded and analyzed using a Mouse Spontaneous Activity Video Analysis System (JLBehv-LAG-4, Shanghai Jiliang Software Technology Co, Ltd).

\section{Chronic social defeat stress (CSDS) induction and behavioral testing}

The CSDS model and social interaction test were performed according to published protocols ${ }^{[32,33]}$. In brief, after screening to identify aggressive CD- 1 mice, the test C57BL/ 6 mice were exposed to a different $\mathrm{CD} 1$ aggressor mouse each day for $10 \mathrm{~min}$ over a total of $10 \mathrm{~d}$. During this exposure, all of the test mice showed signs of stress and subordination, including vocalization, flight response and a submissive posture. After $10 \mathrm{~min}$ of contact, the test mice were separated from the aggressor. The test mice were placed in an adjacent compartment of the same cage, separated by a plastic divider with holes, where they were exposed to chronic stress in the form of threat for the next $24 \mathrm{~h}$. The control mice were housed in equivalent cages but with members of the same strain, which changed daily. Twenty-four hours after the last session, we tested the behavioral consequences of the chronic social defeat stress and selected all of the susceptible mice according to the published method ${ }^{[32]}$. Briefly, the social interaction ratio (SI ratio) was obtained by dividing the time spent in the interaction zone when the target was present by the time spent in the interaction zone when the target was absent. An SI ratio of less than 1 , indicating that less time was spent in the presence versus absence of a social aggressor, was used as the threshold for identifying susceptible mice. All mice were then housed individually for 3 weeks, and all of the 21 susceptible mice were randomly divided into three groups: the DEFEAT group was administered $0.5 \%$ CMC-Na (ig) per day, whereas the YY-23 group was treated with $20 \mathrm{mg} / \mathrm{kg}$ YY-23 (ig) per day, 
and the FLX group was treated with $10 \mathrm{mg} / \mathrm{kg}$ fluoxetine (ip) per day. Eight control mice were administered 0.5\% CMC-Na (ig) per day. We obtained the SI ratio weekly using the same method as previously described.

\section{Statistical analysis}

The data are expressed as the mean \pm SEM. GraphPad Prism5.0 software (for Mac, US) was used to carry out the statistical analysis, and a value of $P<0.05$ was considered to be significant. For the electrophysiological studies, Student's two-tailed t-test was used to determine statistical significance. The $\mathrm{EC}_{50}$ values for NMDA and the $\mathrm{IC}_{50}$ values for $\mathrm{YY}-23$ were obtained using GraphPad Prism 5.0 software (for Mac, US). For the tail suspension test, sucrose preference test, open field test and social interaction test, statistical significance was evaluated by two-way repeat measures ANOVA, and post-hoc analyses were performed with the Bonferroni test.

\section{Results}

YY-23 selectively inhibited NMDA-induced current and showed features as a non-competitive antagonist

For the purpose of investigating the target mechanism of YY-23, an NMDA current was induced on dissociated hippocampal CA1 neurons using a method previously described ${ }^{[25,26]}$, with modifications. The application of 3 $\mu \mathrm{mol} / \mathrm{L}$ YY-23 reduced the NMDA-induced current by more than $50 \%$ (Figure 2C, $n=5, P<0.01$ vs CON); we observed no effect on the holding current and complete recovery by washing (Figure 2A). The dose-response curve of YY-23 gave an
$\mathrm{IC}_{50}$ value of $2.8 \mu \mathrm{mol} / \mathrm{L}$, and the $95 \%$ confidence intervals ranged from 1.8 to $4.2 \mu \mathrm{mol} / \mathrm{L}$ (Figure 2B). In contrast, no effect of the same concentration was detected on the current induced by $50 \mu \mathrm{mol} / \mathrm{L}$ AMPA (Figure $1 \mathrm{~A}$ and $1 \mathrm{C}, n=7$, $P>0.05$ vs $\mathrm{CON})$. Next, we examined the inhibitory effect of YY-23 on different concentrations of NMDA (Figure 3A). The NMDA dose-response curve was constructed in the presence and absence of $3 \mu \mathrm{mol} / \mathrm{L}$ YY-23. The pooled data from four neurons demonstrated that the maximal current response to NMDA showed a $40 \%-50 \%$ reduction in the presence of $3 \mu \mathrm{mol} / \mathrm{L}$ YY-23 (Figure 3B). We also found no prominent change in the $\mathrm{EC}_{50}(46.7 \pm 6.70 \mu \mathrm{mol} / \mathrm{L}$ in the control mice; 49.9 $\pm 9.47 \mu \mathrm{mol} / \mathrm{L}$ with $\mathrm{YY}-23, n=4, P=0.90$ ) (Figure 3C), illustrating the non-competitive antagonist characteristic of $Y Y-23$.

YY-23 inhibited NMDA-induced current in a voltage-independent or 'use-independent' manner

To explore the antagonistic manner of $Y Y-23$, we examined the effects of membrane potential on YY-23 inhibition. The current induced by NMDA was detected in the presence and absence of YY-23 when the membrane potential of the neuron was held at $-30,-60$ and $-90 \mathrm{mV}$ (Figure 4A). The application of $3 \mu \mathrm{mol} / \mathrm{L}$ YY-23 inhibited the NMDA-induced currents equivalently at three different holding potentials, and similar results were observed in five neurons (Figure 4B). We observed no significant differences among groups (Oneway ANOVA, $\left.F_{(2,14)}=0.038, P=0.962\right)$, indicating that the $Y Y-23$ inhibitory effect on the NMDA-induced current was voltageindependent.
A

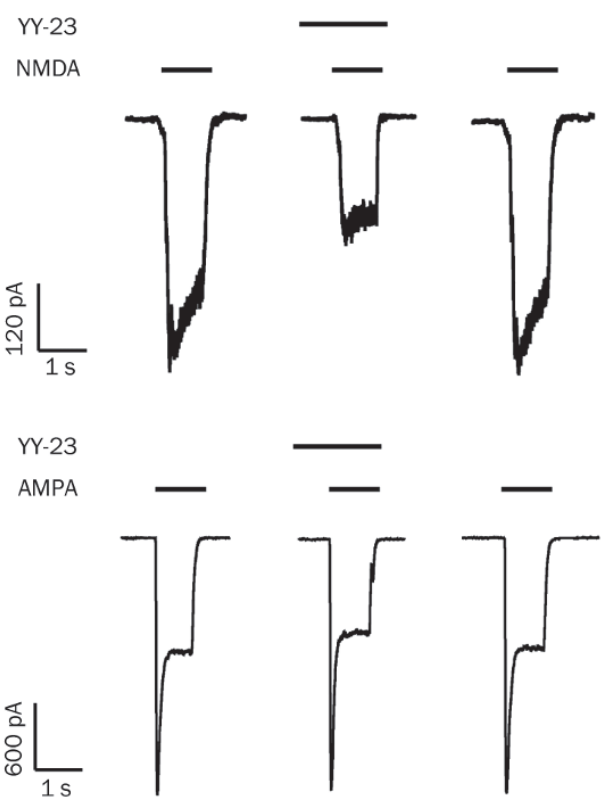

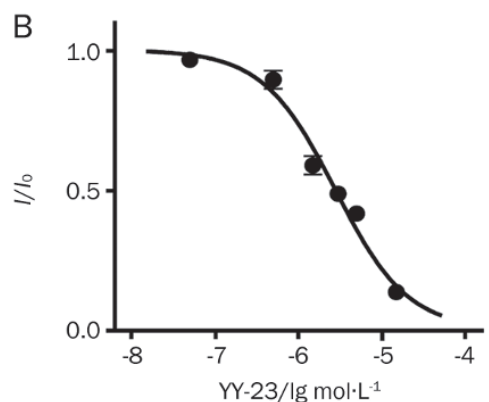

C

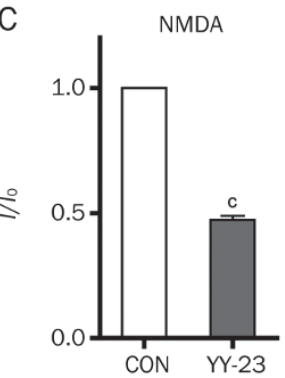

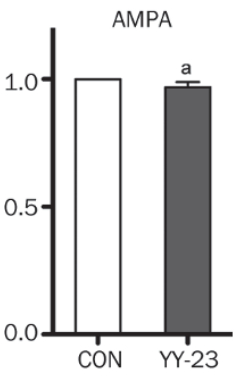

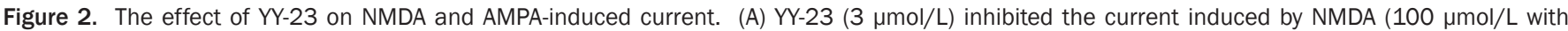
glycine $2 \mu \mathrm{mol} / \mathrm{L})$ but had little effect on the current induced by AMPA $(50 \mu \mathrm{mol} / \mathrm{L})$ of representative neurons. The inserted long and short horizontal lines represent the presence of YY-23 and NMDA (or AMPA), respectively. (B) The dose-response curve of the inhibitory effect of YY-23 on NMDAinduced current; 5-6 neurons were detected for every concentration. (C) The statistical diagram of the inhibitory effect of $3 \mu \mathrm{mol} / \mathrm{L} \mathrm{YY}-23$ on NMDA or AMPA-induced current. The data are represented as the mean \pm SEM. $n=6$ per group. ${ }^{a} P>0.05,{ }^{c} P<0.01$ vs $\operatorname{CON}$. 
To further understand whether the blocking effect of YY-23 on the NMDA receptor was 'agonist-dependent' or 'usedependent,' we examined whether the NMDA-induced currents could be affected by the sustained stimulation of 3 $\mu \mathrm{mol} / \mathrm{L}$ YY-23. One representative test of six repetitions was applied, as shown in Figure 4C. The results showed that the inhibitory strength with rapid onset was almost the same with repeated applications of YY-23 and vanished immediately following the washout of YY-23, indicating that the YY-23 inhibition of the NMDA-induced current was not occurring in a 'use-dependent' manner.

The antagonistic effect of YY-23 on the NMDA receptor was partially blocked by MK-801 and $\mathrm{Mg}^{2+}$

In an effort to understand YY-23's binding characteristics and to confirm the NMDA receptor antagonistic effect of YY-23, the classic antagonist, dizocilpine (MK-801), and the endogenous blocker, $\mathrm{Mg}^{2+}$, were administered, respectively ${ }^{[34]}$. Dosages of $120 \mathrm{nmol} / \mathrm{L}$ for MK-801 and $0.5 \mathrm{mmol} / \mathrm{L}$ for $\mathrm{Mg}^{2+}$

A

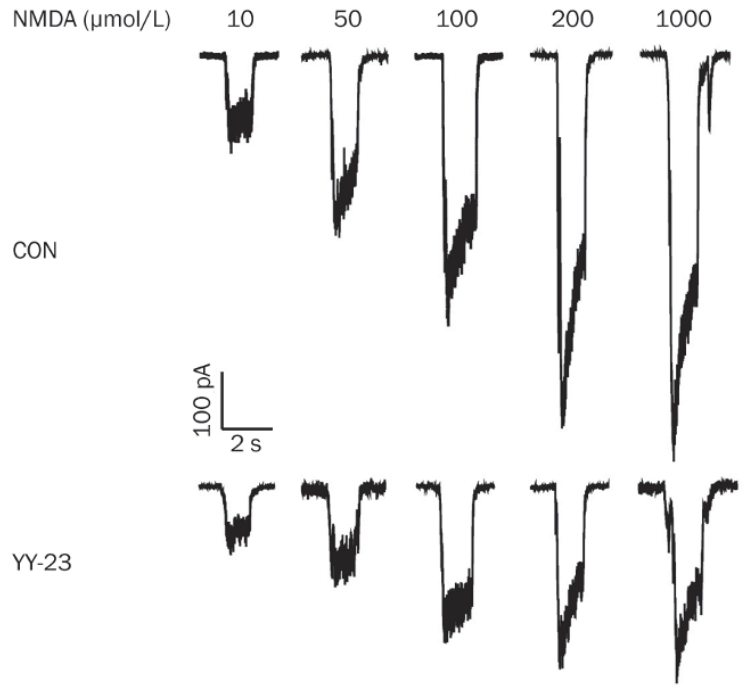

B

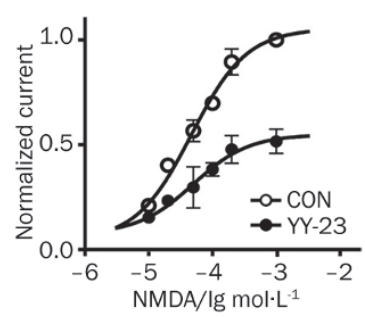

C

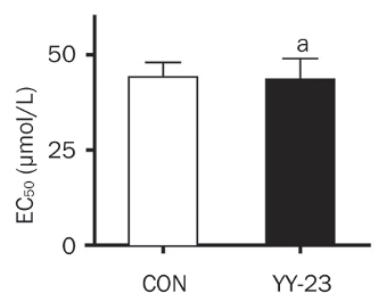

Figure 3. YY-23 showed features as a non-competitive antagonist of the NMDA receptor. (A) YY-23 (3 $\mu \mathrm{mol} / \mathrm{L})$ inhibited the currents induced by different concentrations of NMDA with different extents in a representative neuron. (B) The dose-response curve of normalized pooled data was constructed from the results of four neurons, and the maximal current showed approximately 50\% reduction in the presence of YY-23 $(3 \mu \mathrm{mol} / \mathrm{L})$. (C) No $E_{50}$ of NMDA shifting occurred in the presence of $Y Y-23(3 \mu \mathrm{mol} / \mathrm{L})$, $n=4 .{ }^{a} P>0.05$ vs CON. were selected as working concentrations, based on previous studies $^{[34-36]}$. The results suggested an evident reduction in the NMDA-induced current under these conditions (Figures 5A and $5 \mathrm{~B})$. In the presence of MK-801 and $\mathrm{Mg}^{2+}$, the inhibitory effect of $3 \mu \mathrm{mol} / \mathrm{L}$ YY-23 on the NMDA-induced current was attenuated and found to be significantly less than the inhibitory effect of $3 \mu \mathrm{mol} / \mathrm{L} \mathrm{YY-23}$ alone, which elicited more than a $50 \%$ reduction (Figure 5C). These results demonstrated that the proven blockers such as MK- 801 and $\mathrm{Mg}^{2+}$ could partially neutralize the antagonistic effect of YY-23 on the NMDA receptor, proving the NMDA receptor antagonist activity of YY-23.

YY-23 exhibited a prominent antidepressant-like effect on CMS and a social defeat depression model with rapid onset

Because many types of NMDA receptor antagonists or modulators exhibit prominent antidepressant-like effects with rapid onset, we investigated the psychotropic outcomes of YY-23 as a potentially effective agent against affective disorders. Two animal models were used to validate its efficacy on different aspects of mood disorders. First, an unpredictable CMS model was established using the method described in previ-
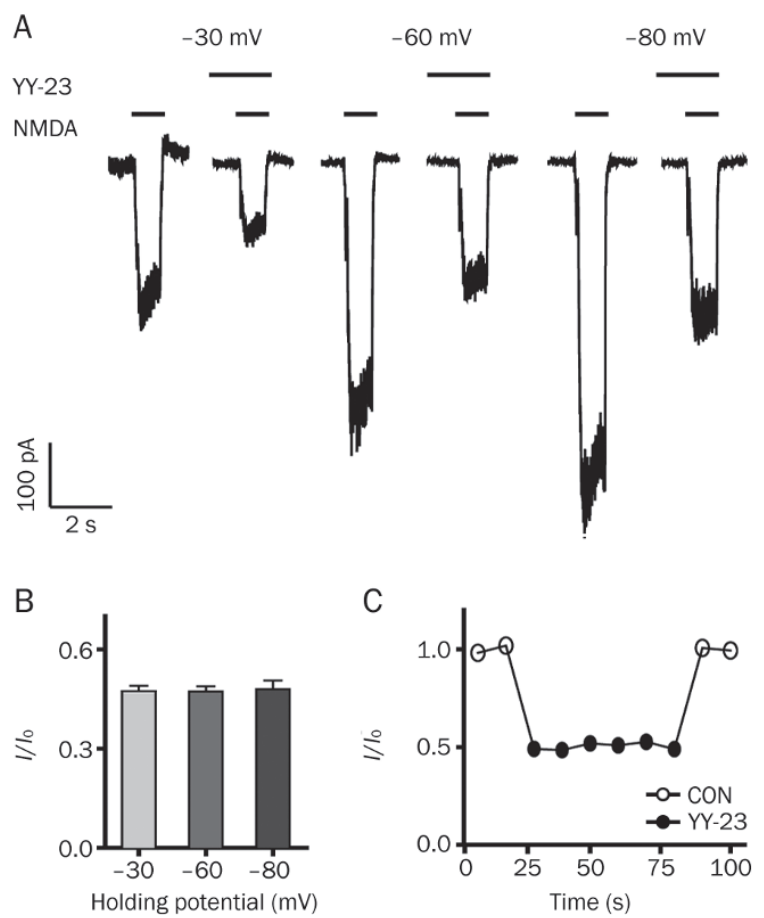

Figure 4. Effects of holding potential and the repeated application of NMDA on the antagonistic effect of YY-23. (A) The effect of YY-23 (3 $\mu \mathrm{mol} / \mathrm{L}$ ) on the NMDA-induced current was tested at three different holding potentials, $-30 \mathrm{mV},-60 \mathrm{mV}$ and $-90 \mathrm{mV}$. The inserted long and short horizontal lines represent the presence of YY-23 and NMDA. (B) The inhibitory effect of YY-23 ( $3 \mu \mathrm{mol} / \mathrm{L}$ ) on NMDA receptors showed no difference among three tested membrane potentials. $n=5$. $P>0.05$. (C) The inhibitory effect of YY-23 (3 $\mu \mathrm{mol} / \mathrm{L})$ on the NMDA receptor did not exhibit use-dependency. Plots of $I / I_{0}$ against a series of current response to NMDA were induced every $10 \mathrm{~s}$ in a representative neuron. 
A
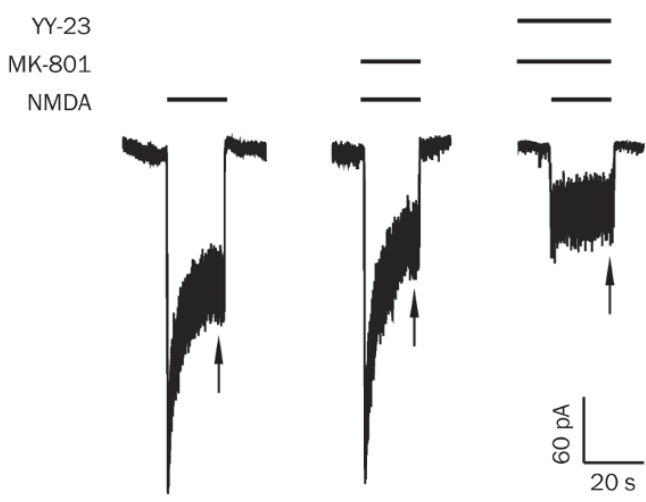

B

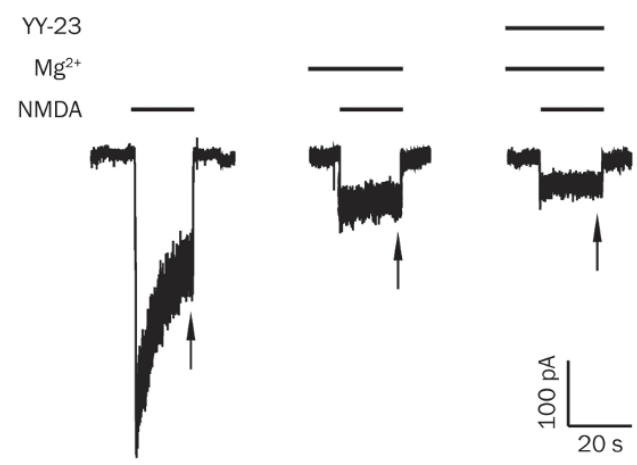

C

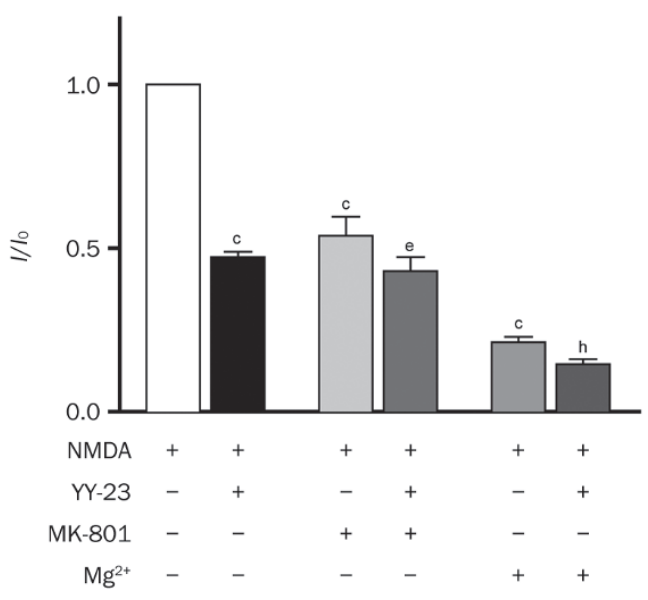

Figure 5. The NMDA receptor antagonistic effect of YY-23 was partially neutralized by proven blockers. (A) The NMDA-induced current of one of the representative neuron showed a reduction after the sustained application of $120 \mathrm{nmol} / \mathrm{L}$ MK-801, and the antagonistic effect of YY-23 (3 $\mu \mathrm{mol} / \mathrm{L}$ ) was partially neutralized by pre-blocked MK-801. (B) The NMDAinduced current of one of the representative neuron exhibited reduction after pre-applied $0.5 \mathrm{mmol} / \mathrm{L} \mathrm{Mg}^{2+}$, partially blocking the antagonistic effect of $Y Y-23$ (3 $\mu \mathrm{mol} / \mathrm{L})$. The inserted long and short horizontal lines in $(A)$ and $(B)$ represent the presence of $Y Y-23, M K-801, \mathrm{Mg}^{2+}$ and NMDA. The steady status of NMDA current is noted by arrows in (A) and (B). (C) Different combinations of applied drugs inhibited the NMDA-induced current to different extents. The ' + ' and '-' below the statistical diagram represent the presence and absence of drugs and NMDA. The data are represented as the mean \pm SEM, ${ }^{c} P<0.01$ vs CON (only NMDA was applied). ${ }^{\mathrm{e}} \mathrm{P}<0.05$ vs MK-801 (NMDA and MK-801 were applied). ${ }^{\mathrm{h}} \mathrm{P}<0.05 \mathrm{vs} \mathrm{Mg}^{2+}$ (NMDA and $\mathrm{Mg}^{2+}$ were applied). $n=5-7$ neurons for every combination of applied drugs. ous studies ${ }^{[27,28,37]}$, followed by behavioral measures of anhedonia (sucrose preference test) and behavioral despair (tail suspension test). A dosage of $20 \mathrm{mg} \cdot \mathrm{kg}^{-1} \cdot \mathrm{d}^{-1}$ was chosen as the optimal dose of YY-23 for the entire experiment based on our preliminary experiments ${ }^{[24]}$. Figures $6 \mathrm{~A}$ and $6 \mathrm{~B}$ show the results of the tail suspension test (TST) and sucrose preference test (SPT) on mice before and after the 6-week CMS treatment. Two-way RM ANOVA revealed the significant effects of treatment $\left(F_{(3,22)}=17.08, P<0.0001\right)$ and time $\left(F_{(3,66)}=18.51, P<0.0001\right)$ on the results of the TST, as well as the significant effects of treatment $\left(F_{(3,22)}=4.36, P=0.0149\right)$ and time $\left(F_{(3,66)}=67.95\right.$, $P<0.0001)$ on the results of the SPT. Once stressed mice exhibited depressive-like behaviors (week 0) after a 3-week CMS induction (post hoc Bonferroni test, all $P<0.001$ vs $\mathrm{CON}$ and $P>0.05$ vs CMS in TST, while in SPT, $P<0.01$ vs CON for CMS, $P<0.001$ vs CON for $Y Y-23, P<0.001$ vs CON for FLX and all $P>0.05$ vs CMS), chronic drug administration was initiated. As shown by Figures 6A and 6B, CMS-induced depressive behaviors were significantly reversed by 3 weeks of treatment with YY-23 and fluoxetine. Furthermore, YY-23 exhibited effects 1 week earlier than treatment with FLX. Specifically, a post hoc Bonferroni test indicated that the immobility time of TST for the YY-23 groups ( $P>0.05$ vs CON, $P<0.001$ vs CMS) had returned to normal at week 2 , whereas the CMS $(P<0.001$ vs $\mathrm{CON})$ and FLX $(P<0.001$ vs $\mathrm{CON}$ and $P>0.05$ vs $\mathrm{CMS})$ groups still showed distinct behavioral despair compared with the vehicle group. At week 3, all of the treatment groups (all $P>0.05$ vs $\mathrm{CON}$ and $P<0.001$ vs CMS) exhibited the successful reversal of immobility. In SPT, the sucrose consumption ratios for the stressed groups still showed low sucrose preference during the first 2 weeks (all $P<0.01$ vs CON and $P>0.05$ vs CMS for week 2) compared with the control group. At week 3, all of the treatment groups, including YY-23 and FLX (all $P>0.05$ vs $\mathrm{CON}, P<0.05$ vs CMS for $\mathrm{YY}-23$, and $P<0.01$ vs CMS for FLX), exhibited successful reversal of anhedonia. To test the possibility that the changed immobility of the stressed mice may be caused by a change in locomotor activity ${ }^{[30]}$, we performed the OFT. Figures 6C and 6D showed that there were no significant effects of treatment $\left(F_{(3,22)}=0.09703, P=0.9608\right)$ and time $\left(F_{(3,66)}=0.01570, P=0.9973\right)$ on total distance, as well as no significant effects of treatment $\left(F_{(3,22)}=0.1618, P=0.9209\right)$ and time $\left(F_{(3,66)}=0.2776, P=0.8414\right)$ on walking speed, suggesting that locomotor activity was virtually normal.

Social avoidance is also a major symptom of mood-related disorders, and social defeat stress mimics several pathological dimensions of depression ${ }^{[32,33,38]}$. Therefore, to study the effect of chronic YY-23 treatment on social interaction behavior, we established the CSDS mouse model. Mice were subjected to chronic defeat stress for 10 consecutive days, followed by a 3-week treatment with YY-23 and FLX. Two-way RM ANOVA revealed a significant effect of treatment $\left(F_{(3,25)}=49.69\right.$, $P<0.0001)$ but no significant effect of time $\left(F_{(3,75)}=0.34\right.$, $P=0.7998)$. As shown in Figure $6 \mathrm{E}$, the defeated mice spent less than $50 \%$ of their time in the interaction zone when an aggressor was introduced into the cage, exhibiting a social interaction ratio (SI ratio) less than 1 (week 0 ), compared with 

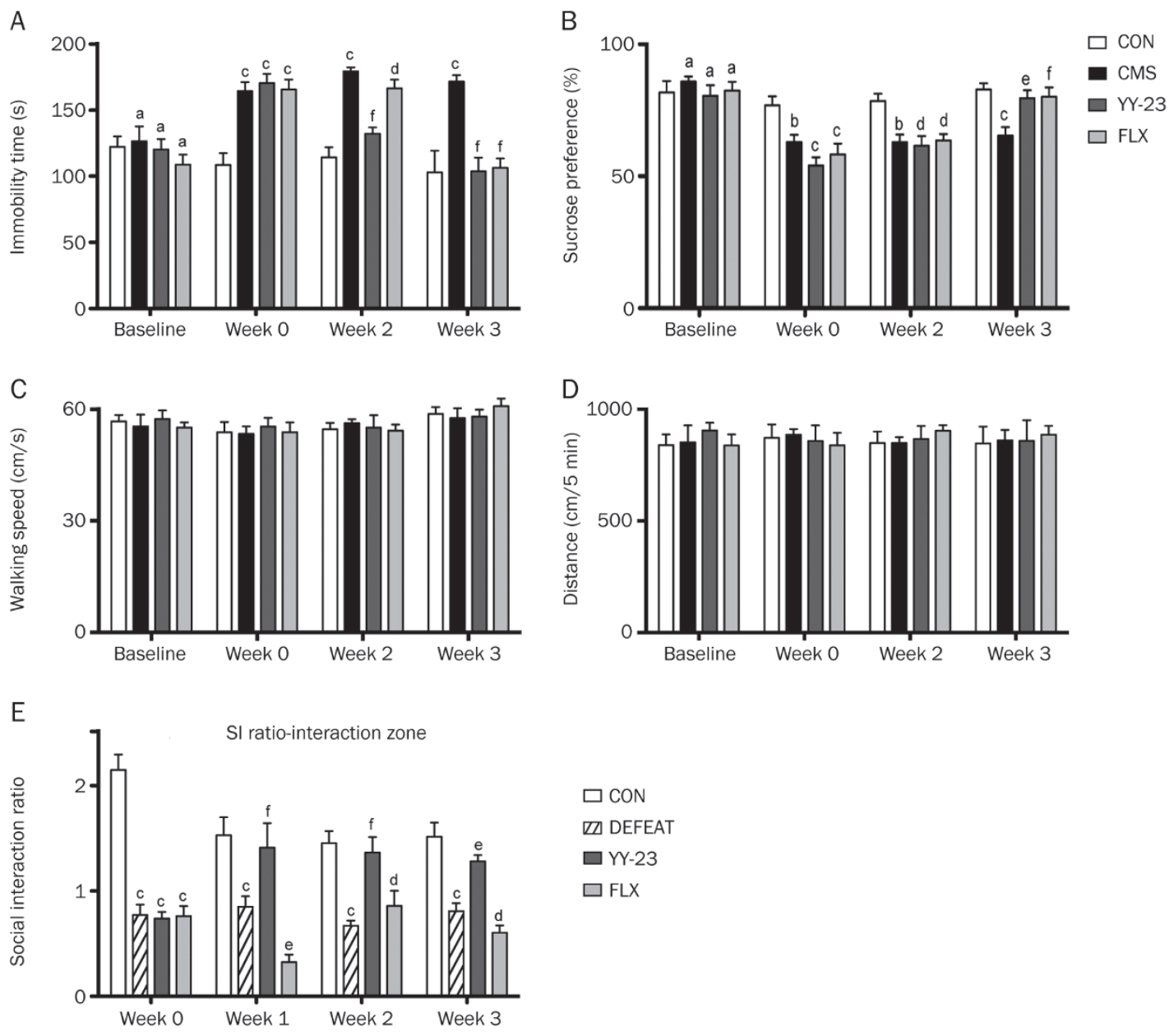

Figure 6. YY-23 reversed the depressive-like behavior induced by CMS and CSDS with rapid onset. (A) YY-23 and fluoxetine (FLX) reversed the immobility deficit of TST induced by CMS stimulation, YY-23 showed an earlier onset than FLX by 1 week. (B) YY-23 and FLX reversed the decreased sucrose consumption in SPT induced by CMS in 3 weeks. Parameters of the immobility in TST and the sucrose consumption in SPT were measured each week. The result of week 1 is not shown. (C) All of the mice exhibited normal locomotor activity represented by walking speed in open field test. (D) All of the mice exhibited normal locomotor activity represented by total distance walked in OFT. (E) YY-23 reversed the decreased social interaction ratio caused by CSDS within 1 week, whereas FLX failed to reverse the social deficit in 3 weeks. Data were represented as the mean \pm SEM. ${ }^{a} P>0.05$, ${ }^{\mathrm{b}} P<0.05,{ }^{\mathrm{c} P}<0.01$ vs CON; ${ }^{\mathrm{d}} P>0.05,{ }^{\mathrm{e}} P<0.05,{ }^{\mathrm{f}} P<0.01$ vs CMS (or DEFEAT).

the control mice (post hoc Bonferroni test, all $P<0.001$ vs CON and $P>0.05$ vs DEFEAT). These promising results showed that a 3-week treatment with YY-23 significantly reversed this social avoidance behavior. In addition, YY-23 triggered the rapid onset of an antidepressant-like effect, causing a reversal in the SI ratio at week 1 (post hoc Bonferroni test, all $P>0.05$ vs CON, $P<0.01$ vs DEFEAT for week $1, P<0.001$ vs DEFEAT for week $2, P<0.05$ vs DEFEAT for week 3 ), whereas FLX failed to exert a reversal effect on the SI ratio within 3 weeks $(P<0.001$ vs CON for week 1 and week $3, P<0.01$ vs CON for week 2, $P<0.05$ vs DEFEAT for week $1, P>0.05$ vs DEFEAT for week 2 and week 3 ).

These results suggested that YY-23 could act as a potential antidepressant, significantly reversing depressive behavior in both CMS and CSDS depression models and exhibiting faster onset than FLX treatment.

\section{Discussion}

YY-23 acts as a non-competitive NMDA receptor antagonist with fast blocking and unblocking characteristics

In the present study, YY-23 acted as an NMDA receptor antagonist and showed no effect on AMPA-induced current at a concentration demonstrated a remarkable inhibition of NMDAinduced current. Therefore, YY-23 could act as a selective NMDA receptor antagonist. The NMDA dose-response curve indicated that in the presence of $Y Y-23$, the maximal response to NMDA was reduced by $40 \%-50 \%$, with little change in the $\mathrm{EC}_{50}$. This result suggested a non-competitive feature of YY-23, indicating that it acted on the allosteric modulatory site of the NMDA receptors, rather than on the glutamate recognition site. Furthermore, the inhibitory effect of $Y Y-23$ on the NMDA-induced current was not affected by membrane potential, and sustained administration of YY-23 inhibited 
all subsequent responses to the same extent and returned to control levels following the washout of YY-23. These results revealed that the inhibition of the NMDA-induced current by YY-23 showed neither 'voltage-dependency' nor 'use-dependency' and demonstrated that YY-23 exhibited the binding feature of fast blocking and unblocking. This suggested that YY-23 did not act at a site within the NMDA receptor-channel lumen ${ }^{[25,34]}$. This is entirely in contrast to the findings for the non-competitive antagonist, ketamine, as well as the uncompetitive channel blockers, memantine and MK- $801^{[25,34,39-42]}$. MK-801 is poorly tolerated clinically due to its slow unblocking kinetics ${ }^{[34,43]}$. YY-23 would be expected to be safer for clinical use because it exhibits fast action with rapid blocking and unblocking rates at low micromolar concentrations.

To validate the direct inhibitory effect of $Y Y-23$ on the NMDA receptor, as shown in Figure 5A, 120 nmol/L MK-801 was applied according to specifications in a previous study ${ }^{[35]}$. The presence of $120 \mathrm{nmol} / \mathrm{L}$ MK-801 partially blocked the inhibitory effect of YY-23 on NMDA-induced current. This result demonstrated that the semi-blocked status of the channel could affect the interaction of YY-23 with the channel. The physiological levels of $\mathrm{Mg}^{2+}(0.5-1 \mathrm{mmol} / \mathrm{L})$ were a natural blocker of the NMDA receptor, causing a substantial reduction in NMDA-induced current at a resting potential ${ }^{[36]}$. In the present study, YY-23 still produced an inhibition of NMDAinduced current in the presence of $0.5 \mathrm{mmol} / \mathrm{L} \mathrm{Mg}^{2+}$, but this was weaker than the level of inhibition that was observed in the presence of $3 \mu \mathrm{mol} / \mathrm{L}$ YY-23 alone (Figure 5B). This result suggested that when $\mathrm{Mg}^{2+}$ produced an ionic blockade of the channel ${ }^{[36]}$, it affected the interaction of YY-23 with the channel. All of these findings suggested that when the channel of the NMDA receptor was blocked by other blockers, such as MK-801 and $\mathrm{Mg}^{2+}$, the gating status of the channel was changed. Based on this, the inhibitory effect of YY-23 on the NMDA receptor could be directly influenced by an altered channel status. Therefore, we can conclude that YY-23 acts directly on the NMDA receptor, rather than through a non-specific effect, or through intracellular signaling mechanisms, illustrating that YY-23 likely acts as an NMDA receptor antagonist.

All of these results demonstrated that YY-23 may act as a selective, non-competitive NMDA receptor antagonist. In addition, YY-23 may be a safer agent than other traditional NMDA receptor antagonists and blockers.

\section{YY-23 showed antidepressant potential with rapid onset in CMS and CSDS models}

Many types of animal models have been developed to imitate the clinical syndrome of depression in patients. An animal model induced by CMS was developed and confirmed to be the most similar to the symptoms of clinical depression due to the construct validity, face validity and predictive validity $^{[28,44,45]}$. In the present study, we investigated the effects of YY-23 and FLX on depressive behavior caused by CMS stressors, including alterations in the day/night cycle, rotated or soiled cages, isolated housing and food/water deprivation. A 3-week chronic treatment with YY-23 and FLX reversed the reduced sensitivity to reward (anhedonia) and the reduced desire to struggle (despair). Beyond that, the most striking finding was that despite the same onset as FLX in anhedonia that was indicated by SPT, YY-23 exhibited the rapid ability to reverse the depressive behavior 1 week earlier than the SSRI FLX, which had a delayed onset of 3 weeks. These results demonstrated that YY-23 elicited an antidepressant effect on a CMS depression model with faster onset than the SSRIs that are widely used clinically.

Social avoidance is also the main symptom of clinical depression ${ }^{[46]}$; moreover, social stressors always control affective-like behavioral responses across a wide variety of mammalian species ${ }^{[32]}$. Therefore, an animal model reflecting a deficit of social behavior is necessary to explore the pathological mechanism of depression and antidepressant action. CSDS is an increasingly popular model that exploits the ethological relevance of territorial aggression. It is considered to have construct, face, and predictive validity ${ }^{[32,47]}$ for the assessment of social interaction. The reversal of defeat-induced social avoidance in mice by antidepressants also suggests that this behavioral pathology may be relevant to human depres-

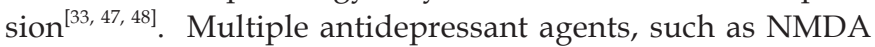
receptor antagonists and standard antidepressants, have been validated as being capable of reversing the social interaction effect of social defeat, but long-term treatment is required for the effectiveness of standard antidepressants ${ }^{[33,48]}$. Therefore, to further understand the antidepressant-like effect of YY-23, we established the CSDS model using a published protocol and explored the effect of YY-23 and FLX on social avoidance in the present study. Our findings showed that YY-23 prominently reversed the decreased social interaction ratio that was induced by social defeat stress, with a very rapid onset of 1 week. However, FLX failed to rescue the deficit in social interaction within 3 weeks. This result was consistent with those of previous studies, in which FLX showed a slow onset time of 4 weeks ${ }^{[33,48]}$. Therefore, these results demonstrated that YY-23 was absolutely effective on social behavior, exerting an antidepressant-like effect in a CSDS model with significantly faster onset than standard antidepressants.

All of these observations provided powerful evidence suggesting that $Y Y-23$, acted as a selective antagonist of NMDA receptor and may be more suitable for use as a rapid-acting antidepressant than the SSRIs with slow onset that are used clinically.

\section{YY-23 possesses a distinct possibility of being a rapid anti- depressant-like agent}

The antidepressants used clinically function primarily by elevating the concentrations of synaptic monoamines, such as serotonin, but are associated with various side effects and a long timescale of action. On this occasion, the NMDA receptor antagonist displayed advantageous characteristics, such as rapid action. Various compounds with NMDA antagonist actions are awaiting testing in clinical trials ${ }^{[11]}$. The hypothesis that NMDA antagonists are effective antidepressants was based on the observation that interfering with NMDA 
antagonists such as MK-801, 1-aminocyclopropanecarboxylic acid (a partial agonist at glycine B receptors) and D,L-2-amino7-phosphonoheptanoic acid (a competitive antagonist) would mitigate the behavioral deficits induced by inescapable stressors in rodents ${ }^{[49,50]}$. Multiple NMDA receptor antagonists were reported to mimic the effects of antidepressants in more complex models, including chronic mild stress and olfactory bulbectomy ${ }^{[51-53]}$. This clinical and preclinical evidence linked major depressive disorder to dysregulated glutamate neurotransmission. In our previous study, similar observations were made. CMS could cause a deficit in spontaneousburst firing in PFC pyramidal cells, and YY-23 treatment may enhance glutamate neurotransmission based on reversing the spontaneous-burst firing and could also rescue the CMSinduced decrease in BDNF levels ${ }^{[24]}$. All of these processes are the pillars of the glutamatergic theory of depression, demonstrating the rapid antidepressant potential of NMDA receptor antagonists. Moreover, the antagonistic effect on the NMDA receptor affords $\mathrm{YY}-23$ the capability to act as a rapid-acting antidepressant.

\section{The possible mechanism involved in the antidepressant-like} effect of YY-23

In recent years, the response to intravenous ketamine infusion has now been replicated in multiple studies, including in depressed patients with suicidal ideation, treatment-resistant depression and bipolar disorder ${ }^{[54,55]}$. The rapid and robust antidepressant effects of the NMDA receptor antagonists ketamine and traxoprodil provided target validation for the family of ionotropic glutamate receptors ${ }^{[10,54]}$. In the present study, we observed that YY-23 could act as a non-competitive NMDA receptor antagonist; moreover, YY-23 indeed exhibited rapid antidepressant-like effects on both CMS and CSDS models. Based on these observations and the research results of other NMDA receptor antagonists, we speculated that some detailed mechanisms may be involved in the antidepressantlike effects of YY-23. With the capacity to block the NMDA receptor, YY-23 could likely enhance neurotransmission by one of two ways. First, if YY-23 blocked the extra-synaptic NMDA receptors, it could reduce signaling via elongation factior-2, thereby disinhibiting BDNF levels ${ }^{[56,57]}$. An alternative possibility is that YY-23 acts on synaptic NMDA receptors, stimulating synaptic glutamate release and enhancing synaptic AMPA receptor downstream signaling ${ }^{[6,8]}$. Both mechanisms lead to dendritic spine growth, followed by enhanced synaptic plasticity, neurotransmission and functional connectivity ${ }^{[56]}$. This speculation that YY-23 may enhance synaptic neurotransmission was not only supported by numerous observations related to the effects of other NMDA receptor antagonists ${ }^{[58-60]}$ but was also confirmed by our previous work, which showed the significant ability of YY-23 to reverse the decreased burst firing induced by $\mathrm{CMS}^{[24]}$. Regarding the downstream mechanism, we have noted interesting changes in some of the signaling pathways downstream of the glutamate receptors after treatment with YY-23, but additional work is needed to draw definitive conclusions regarding these changes.

\section{Conclusions}

The present study highlights that YY-23, which is derived from timosaponin B-III, could act as a selectively non-competitive NMDA receptor antagonist that does not exhibit voltagedependency or use-dependency. Behavioral studies aiming to reveal the psychotropic outcomes of YY-23 noted the rapid antidepressant-like effects on both the CMS and the CSDS models. Although further studies will be necessary to elucidate the neurotrophic molecular expression (or signal transduction pathways) and detailed mechanisms of rapid action by $\mathrm{YY}-23$, these results unveiled the target mechanism of the metabolite of timosaponin B-III and confirmed the essential roles of NMDA receptor antagonists in antidepressant treatments. Moreover, we provided evidence of the therapeutic potential of timosaponin for the treatment of depression and demonstrated the more rapid onset of the effectiveness of the compound compared with SSRIs.

\section{Acknowledgements}

This work was supported by the National Natural Science Foundation (31128009 and 31171011 to Yang LI), the National Science \& Technology Major Project 'Key New Drug Creation and Manufacturing Program' (2014ZX09102-001-005 to Chenggang HUANG), and the Ministry of Science and Technology (2013CB910601 to Yang LI).

\section{Author contribution}

Qi ZHANG performed the electrophysiological experiments; Qi ZHANG, Fei GUO and Bing ZHANG performed the behavioural experiments and analysed the data; Zhi-wen FU and Cheng-gang Huang contributed the new compounds; Qi ZHANG, Cheng-gang HUANG and Yang LI designed the project and wrote the paper.

\section{References}

1 Krishnan V, Nestler EJ. The molecular neurobiology of depression. Nature 2008; 455: 894-902.

2 Belmaker RH, Agam G. Major depressive disorder. N Engl J Med 2008; 358: 55-68.

3 Davidson JR. Major depressive disorder treatment guidelines in America and Europe. J Clin Psychiatry 2010; 71 Suppl E1: e04.

4 Gelenberg AJ. A review of the current guidelines for depression treatment. J Clin Psychiatry 2010; 71: e15.

5 Lapidus KA, Soleimani L, Murrough JW. Novel glutamatergic drugs for the treatment of mood disorders. Neuropsychiatr Dis Treat 2013; 9: 1101-12.

6 Duman RS, Voleti B. Signaling pathways underlying the pathophysiology and treatment of depression: novel mechanisms for rapid-acting agents. Trends Neurosci 2012; 35: 47-56.

7 Larkin GL, Beautrais AL. A preliminary naturalistic study of lowdose ketamine for depression and suicide ideation in the emergency department. Int J Neuropsychopharmacol 2011; 14: 1127-31.

8 Li N, Lee B, Liu RJ, Banasr M, Dwyer JM, Iwata M, et al. mTORdependent synapse formation underlies the rapid antidepressant effects of NMDA antagonists. Science 2010; 329: 959-64.

9 Jourdi H, Hsu YT, Zhou M, Qin Q, Bi X, Baudry M. Positive AMPA receptor modulation rapidly stimulates BDNF release and increases dendritic mRNA translation. J Neurosci 2009; 29: 8688-97. 
10 Preskorn SH, Baker B, Kolluri S, Menniti FS, Krams M, Landen JW. An innovative design to establish proof of concept of the antidepressant effects of the NR2B subunit selective $N$-methyl- $D$-aspartate antagonist, CP-101,606, in patients with treatment-refractory major depressive disorder. J Clin Psychopharmacol 2008; 28: 631-7.

11 Pilc A, Wieronska JM, Skolnick P. Glutamate-based antidepressants: preclinical psychopharmacology. Biol Psychiatry 2013; 73: 1125-32.

12 Murrough JW, Charney DS. Cracking the moody brain: lifting the mood with ketamine. Nat Med 2010; 16: 1384-5.

13 Murrough JW, Charney DS. Is there anything really novel on the antidepressant horizon? Curr Psychiatry Rep 2012; 14: 643-9.

14 Reus GZ, Stringari RB, Ribeiro KF, Ferraro AK, Vitto MF, Cesconetto P, et al. Ketamine plus imipramine treatment induces antidepressantlike behavior and increases CREB and BDNF protein levels and PKA and PKC phosphorylation in rat brain. Behav Brain Res 2011; 221: 166-71.

15 Neis VB, Moretti M, Manosso LM, Lopes MW, Leal RB, Rodrigues AL. Agmatine enhances antidepressant potency of MK-801 and conventional antidepressants in mice. Pharmacol Biochem Behav 2015; 130: 9-14.

16 Behrens MM, Ali SS, Dao DN, Lucero J, Shekhtman G, Quick KL, et al. Ketamine-induced loss of phenotype of fast-spiking interneurons is mediated by NADPH-oxidase. Science 2007; 318: 1645-7.

17 Zhu WL, Ma SP, Qu R, Kang DL, Liu YD. Antidepressant effect of baicalin extracted from the root of Scutellaria baicalensis in mice and rats. Pharm Biol 2006; 44: 503-10.

18 Ren LX, Luo YF, Li X, Zuo DY, Wu YL. Antidepressant-like effects of sarsasapogenin from Anemarrhena asphodeloides BUNGS (Liliaceae). Biol Pharma Bull 2006; 29: 2304-6.

19 Wang R, Yan H, Tang XC. Progress in studies of huperzine A, a natural cholinesterase inhibitor from Chinese herbal medicine. Acta Pharmacol Sin 2006; 27: 1-26.

20 Guo Y, Zhang H, Chen X, Cai W, Cheng J, Yang Y, et al. Evaluation of the antipsychotic effect of bi-acetylated I-stepholidine (I-SPD-A), a novel dopamine and serotonin receptor dual ligand. Schizophr Res 2009; 115: 41-9.

21 Matsuda H, Sato N, Yamazaki M, Naruto S, Kubo M. Testosterone 5 alpha-reductase inhibitory active constituents from Anemarrhenae Rhizoma. Biol Pharm Bull 2001; 24: 586-7.

22 Jiang W, Guo J, Xue R, Zhu K, Li Z, Chen M, et al. Anti-depressive activities and biotransformation of timosaponin B-III and its derivatives. Nat Prod Res 2014; 28: 1446-53.

23 Li TJ, Qiu Y, Yang PY, Rui YC, Chen WS. Timosaponin B-II improves memory and learning dysfunction induced by cerebral ischemia in rats. Neurosci Lett 2007; 421: 147-51.

24 Guo F, Zhang Q, Zhang B, Fu Z, Wu B, Huang C, et al. Burst-firing patterns in the prefrontal cortex underlying the neuronal mechanisms of depression probed by antidepressants. Eur J Neurosci 2014; 40: 3538-47.

25 Zhang JM, Hu GY. Huperzine A, a nootropic alkaloid, inhibits $N$-methyl$D$-aspartate-induced current in rat dissociated hippocampal neurons. Neuroscience 2001; 105: 663-9.

26 Sodickson DL, Bean BP. Neurotransmitter activation of inwardly rectifying potassium current in dissociated hippocampal CA3 neurons: interactions among multiple receptors. J Neurosci 1998; 18: 815362.

27 Willner P. The validity of animal models of depression. Psychopharmacology (Berl) 1984; 83: 1-16.

28 Willner P. Chronic mild stress (CMS) revisited: consistency and behavioural-neurobiological concordance in the effects of CMS. Neuropsychobiology 2005; 52: 90-110.
29 Ageta H, Murayama A, Migishima R, Kida S, Tsuchida K, Yokoyama $\mathrm{M}$, et al. Activin in the brain modulates anxiety-related behavior and adult neurogenesis. PLoS One 2008; 3: e1869.

30 Taniguchi S, Nakazawa T, Tanimura A, Kiyama Y, Tezuka T, Watabe $\mathrm{AM}$, et al. Involvement of NMDAR2A tyrosine phosphorylation in depression-related behaviour. EMBO J 2009; 28: 3717-29.

31 Stuart MJ, Corrigan F, Baune BT. Knockout of CXCR5 increases the population of immature neural cells and decreases proliferation in the hippocampal dentate gyrus. J Neuroinflammation 2014; 11: 31.

32 Golden SA, Covington HE, 3rd, Berton O, Russo SJ. A standardized protocol for repeated social defeat stress in mice. Nat Protoc 2011; 6: 1183-91.

33 Tsankova NM, Berton O, Renthal W, Kumar A, Neve RL, Nestler EJ. Sustained hippocampal chromatin regulation in a mouse model of depression and antidepressant action. Nat Neurosci 2006; 9: 51925.

34 Gilling KE, Jatzke C, Hechenberger M, Parsons CG. Potency, voltagedependency, agonist concentration-dependency, blocking kinetics and partial untrapping of the uncompetitive $N$-methyl- $D$-aspartate (NMDA) channel blocker memantine at human NMDA (GIuN1/GIuN2A) receptors. Neuropharmacology 2009; 56: 866-75.

35 Frankiewicz T, Potier B, Bashir ZI, Collingridge GL, Parsons CG. Effects of memantine and MK-801 on NMDA-induced currents in cultured neurones and on synaptic transmission and LTP in area CA1 of rat hippocampal slices. Br J Pharmacol 1996; 117: 689-97.

36 Huettner JE, Bean BP. Block of $N$-methyl-D-aspartate-activated current by the anticonvulsant MK-801: selective binding to open channels. Proc Natl Acad Sci U S A 1988; 85: 1307-11.

37 Willner P. Validity, reliability and utility of the chronic mild stress model of depression: a 10-year review and evaluation. Psychopharmacology (Berl) 1997; 134: 319-29.

38 Fuchs E, Flugge G. Social stress in tree shrews: effects on physiology, brain function, and behavior of subordinate individuals. Pharmacol Biochem Behav 2002; 73: 247-58.

39 Johnson JW, Kotermanski SE. Mechanism of action of memantine. Curr Opin Pharmacol 2006; 6: 61-7.

40 LePage KT, Ishmael JE, Low CM, Traynelis SF, Murray TF. Differential binding properties of $\left[{ }^{3} \mathrm{H}\right]$ dextrorphan and $\left[{ }^{3} \mathrm{H}\right] \mathrm{MK}-801$ in heterologously expressed NMDA receptors. Neuropharmacology 2005; 49: 1-16.

41 Parsons CG, Gruner R, Rozental J, Millar J, Lodge D. Patch clamp studies on the kinetics and selectivity of $N$-methyl- $D$ aspartate receptor antagonism by memantine (1-amino-3,5dimethyladamantan). Neuropharmacology 1993; 32: 1337-50.

42 Parsons CG, Danysz W, Quack G. Memantine is a clinically well tolerated $N$-methyl-D-aspartate (NMDA) receptor antagonist - a review of preclinical data. Neuropharmacology 1999; 38: 735-67.

43 McKay S, Bengtson CP, Bading H, Wyllie DJ, Hardingham GE. Recovery of NMDA receptor currents from MK-801 blockade is accelerated by $\mathrm{Mg}^{2+}$ and memantine under conditions of agonist exposure. Neuropharmacology 2013; 74: 119-25.

44 Gambarana C, Scheggi S, Tagliamonte A, Tolu P, De Montis MG. Animal models for the study of antidepressant activity. Brain Res Brain Res Protoc 2001; 7: 11-20.

45 Forbes NF, Stewart CA, MAtthews K, Reid IC. Chronic mild stress and sucrose consumption: Validity as a model of depression. Physiol Behav 1996; 60: 1481-4.

46 Nestler EJ, Hyman SE. Animal models of neuropsychiatric disorders. Nat Neurosci 2010; 13: 1161-9.

47 Donahue RJ, Muschamp JW, Russo SJ, Nestler EJ, Carlezon WA, Jr. Effects of striatal DeltaFosB overexpression and ketamine on social 
defeat stress-induced anhedonia in mice. Biol Psychiatry 2014; 76 : 550-8.

48 Berton O, McClung CA, Dileone RJ, Krishnan V, Renthal W, Russo SJ, et al. Essential role of BDNF in the mesolimbic dopamine pathway in social defeat stress. Science 2006; 311: 864-8.

49 Trullas R, Skolnick P. Functional antagonists at the NMDA receptor complex exhibit antidepressant actions. Eur J Pharmacol 1990; 185: $1-10$.

50 Skolnick P, Popik P, Trullas R. Glutamate-based antidepressants: 20 years on. Trends Pharmacol Sci 2009; 30: 563-9.

51 Papp M, Moryl E. New evidence for the antidepressant activity of MK-801, a non-competitive antagonist of NMDA receptors. Pol J Pharmacol 1993; 45: 549-53.

52 Papp M, Moryl E. Antidepressant activity of non-competitive and competitive NMDA receptor antagonists in a chronic mild stress model of depression. Eur J Pharmacol 1994; 263: 1-7.

53 Redmond AM, Harkin A, Kelly JP, Leonard BE. Effects of acute and chronic antidepressant administration on phencyclidine (PCP) induced locomotor hyperactivity. Eur Neuropsychopharmacol 1999; 9: 165-70.

54 Zarate CA Jr, Singh JB, Carlson PJ, Brutsche NE, Ameli R, Luckenbaugh $\mathrm{DA}$, et al. A randomized trial of an $\mathrm{N}$-methyl-D-aspartate antagonist in treatment-resistant major depression. Arch Gen Psychiatry 2006; 63:
856-64.

55 Diazgranados N, Ibrahim L, Brutsche NE, Newberg A, Kronstein P, Khalife S, et al. A randomized add-on trial of an $N$-methyl- $D$-aspartate antagonist in treatment-resistant bipolar depression. Arch Gen Psychiatry 2010; 67: 793-802.

56 Krystal JH, Sanacora G, Duman RS. Rapid-acting glutamatergic antidepressants: the path to ketamine and beyond. Biol Psychiatry 2013; 73: 1133-41.

57 Autry AE, Adachi M, Nosyreva E, Na ES, Los MF, Cheng PF, et al. NMDA receptor blockade at rest triggers rapid behavioural antidepressant responses. Nature 2011; 475: 91-5.

58 Stone JM, Dietrich C, Edden R, Mehta MA, De Simoni S, Reed L, et al. Ketamine effects on brain GABA and glutamate levels with ${ }^{1} \mathrm{H}$-MRS: relationship to ketamine-induced psychopathology. Mol Psychiatry 2012; 17: 664-5.

59 Krystal JH, D'Souza DC, Mathalon D, Perry E, Belger A, Hoffman R. NMDA receptor antagonist effects, cortical glutamatergic function, and schizophrenia: toward a paradigm shift in medication development. Psychopharmacology (Berl) 2003; 169: 215-33.

60 Chowdhury GM, Behar KL, Cho W, Thomas MA, Rothman DL, Sanacora G. ${ }^{1} \mathrm{H}-\left[{ }^{13} \mathrm{C}\right]$-nuclear magnetic resonance spectroscopy measures of ketamine's effect on amino acid neurotransmitter metabolism. Biol Psychiatry 2012; 71: 1022-5. 